\title{
Rapid Analytic Determination of Dry Cask Storage Canister Internal Temperatures
}

\author{
Evan T. Palmer ${ }^{1}$ and Matthew M. Barry ${ }^{2}$ \\ Department of Mechanical Engineering and Materials Science, University of Pittsburgh, Pittsburgh, PA, USA. \\ 1etp18@pitt.edu and ${ }^{2} m m b 49 @ p i t t . e d u$
}

\section{INTRODUCTION}

In the absence of a permanent disposition pathway, the service lifetimes of spent fuel dry cask storage systems (DCS) will likely be longer than originally intended [1]. Due to the still-radioactive nature of the spent fuel, a breach of containment, and the subsequent environmental remediation, would pose a serious hazard to public health. As most canister degradation mechanisms are temperature-dependent, it is essential to develop tools and methods to identify canisters at risk of breach due to prolonged thermal exposure [1].There is currently no expedient method to physically monitor the thermal environment within the DCS. Consequently, a number of studies have modeled temperature profiles of a given DCS configuration with a unique fuel loading exposed to a singular environmental condition using commercially available computational fluid dynamics (CFD) software [2, 3, 4]. Given the large number of individual storage modules, each with a unique fuel activity, the need to predict realistic temperature profiles as a function of time cannot be met through use of CFD modeling due to the computational expense. The purpose of this study is to demonstrate that a low-fidelity, yet robust analytic model can accurately determine these temperate distributions.

\section{METHODOLOGY}

To analytically model the temperature distribution within a DCS system, a two-dimensional linear thermal resistance network (TRN) was developed. The model was based upon the NUHOMS HSM-1 storage module at Calvert Cliffs Nuclear Power Station Independent Spent Fuel Storage Installation [2]. For brevity, a schematic of the northeast quarter of the DCS is shown in Fig. 1. The TRN was governed by the following relation

$$
q=\frac{\Delta T}{R_{t h}}
$$

where $q$ is the heat generation rate of each fuel assembly as reported in [2], $\Delta T$ is temperature difference between nodes, and $R_{t h}$ is the thermal resistance associated with each component, with component-wise values reported in Tab. I. These inputs then allowed for the resolution of nodal temperature, $T$. The solution to the systems of equations constructed via applying Eqn. 1 to each node of the DCS system is presented in the following.

\section{Mathematical Model}

There are five unique temperatures describing each fuel assembly: a center, north, south, east and west, denoted $T_{i, \mathrm{C}}$, $T_{i, \mathrm{~N}}, T_{i, \mathrm{~S}}, T_{i, \mathrm{E}}$, and $T_{i, \mathrm{~W}}$, respectively, where $i$ is the number of the assembly, ranging from 1 to 24 . This is illustrated for the fifth fuel assembly, as denoted in Fig. 1. Each assembly has a corresponding thermal resistance denoted $R_{\mathrm{asm}, i}$, which is the same in each principal direction, and is shown for the first fuel assembly in Fig. 1. The heat generation term $q_{i}$ is applied at each assembly center temperature node, as shown for the sixth assembly in Fig. 1.

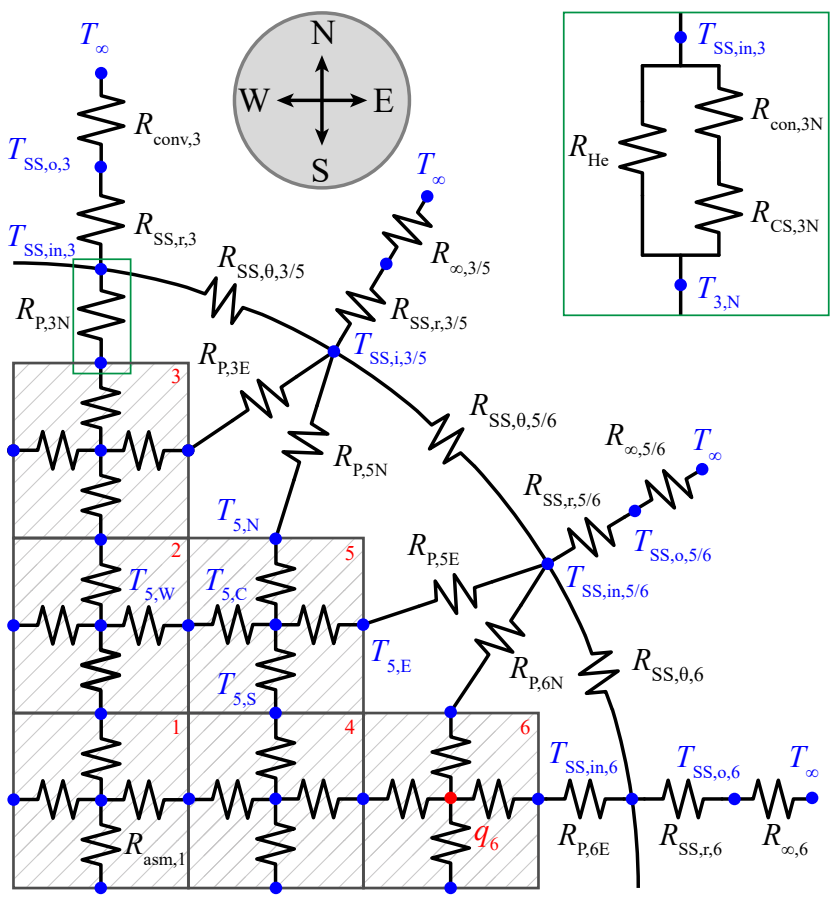

Fig. 1. Schematic of spent fuel assemblies, as indicated by hashmarks, within DCS, with overlaid thermal resistances and nodal temperatures comprising the TRN. Temperatures are denoted by a dot and text in blue coloration. Heat generation is denoted by a dot and text in red coloration. Each assembly number is listed in the top right corner. Inset depicts parallel thermal resistance of $\mathrm{He}$ and CS. Interfacial temperatures between cells are of equal value.

The canister is back-filled with helium (He), and the fuel assemblies are supported by carbon steel (CS) spacer discs. Thus, heat is able to flow from the assemblies to the canister via convection and conduction. Due to the complexity of modeling natural convection between planar and non-planar faces within an enclosure, the present TRN model presents data for a conduction-only heat transfer through the helium domain. The exterior temperature nodes of the third, fifth and sixth assemblies are connected to the interior temperature nodes of the stainless steel (SS) canister, denoted by $T_{\mathrm{SS}, \text { in, } i}$, via parallel resistances, $R_{\mathrm{P}, i}$, with the last subscript indicating direction from the fuel assembly $i$. The parallel resistance is based upon the thermal resistance of the helium, $R_{\mathrm{He}}$ and carbon steel 
spacer disc $R_{\mathrm{CS}, i}$, with the last subscript indicating direction. The carbon steel spacer disc is in series with a thermal contact resistance, $R_{\mathrm{con}, i}$ that varies as a function of angular position, and with the last subscript indicating direction from the fuel assembly. The contact resistance is modeled as,

$$
R_{\mathrm{con}, i}=\frac{L_{\mathrm{gap}}}{k_{\mathrm{CS}} A_{c}}
$$

where $L_{\text {gap }}$ is the spacer gap width, given in [2] and $A_{c}$ is the area normal to the direction of heat flow.

TABLE I. Thermal Resistance Correlations

\begin{tabular}{lll}
\hline Material & $k_{\text {eff }}$ & Expression \\
\hline Fuel Assembly & See [5] & $R_{\mathrm{asm}}=\frac{R_{o}^{2}}{4 k_{\mathrm{eff}} V_{\mathrm{asm}}}$ \\
Helium & See [6] & $R_{\mathrm{He}}=\frac{L_{c}}{k_{\mathrm{He}} A_{c}}$ \\
Carbon Steel & $63\left[\mathrm{~W} / \mathrm{m}^{2} \mathrm{~K}\right]$ & $R_{\mathrm{CS}}=\frac{L_{c}}{k_{\mathrm{CS}} A_{c}}+R_{\mathrm{con}, i}$ \\
Steel/Helium & $\mathrm{N} / \mathrm{A}$ & $R_{\mathrm{eff}}=\frac{R_{\mathrm{CS}} R_{\mathrm{He}}}{R_{\mathrm{CS}}+R_{\mathrm{He}}}$ \\
& & \\
Stainless Steel & $15.1\left[\mathrm{~W} / \mathrm{m}^{2} \mathrm{~K}\right]$ & $R_{\mathrm{SS}, \mathrm{r}}=\frac{\ln \left(\frac{r_{o}}{r_{\mathrm{in}}}\right)}{2 \pi L_{\mathrm{asm}} k_{\mathrm{SS}}}$ \\
& & \\
Stainless Steel ${ }^{a}$ & $15.1\left[\mathrm{~W} / \mathrm{m}^{2} \mathrm{~K}\right]$ & $R_{\mathrm{SS}, c}=\frac{\alpha}{k_{\mathrm{SS}} A_{c}}$ \\
& &
\end{tabular}

${ }^{a} \alpha$ is the arc length between surface temperature nodes.

Heat can conduct radially through the canister, as well as circumferentially. The respective thermal resistances are denoted $R_{\mathrm{SS}, \mathrm{r}, i}$, and $R_{\mathrm{SS}, \theta, i}$. The last number(s) in the subscript indicate between which temperature nodes the heat can conduct. The temperature on the exterior of the canister is denoted $T_{\mathrm{SS}, \mathrm{o}, i}$. The free-stream temperature is denoted as $T_{\infty}$ and is held at invariant at 298 [K]. The thermal resistance associated with convection is denoted as $R_{\infty, i}$. Convection from the surface of the canister to the ambient air was modeled using Newtons Law of Cooling, expressed as

$$
q=h A_{s}\left(T_{\mathrm{SS}, \mathrm{o}, i}-T_{\infty}\right),
$$

where $A_{s}$ is one-sixteenth of the canister's surface area and $h$ is the convective heat transfer coefficient, expressed as,

$$
h=\frac{N u_{\mathrm{eff}} k_{\mathrm{air}}}{D}
$$

$N u_{\text {eff }}$ is the effective Nusselt number, $k_{\text {air }}$ is the thermal conductivity of air, and $D$ is the diameter of the canister. In order to represent the variation in the convective heat transfer coefficient as a function of angular position around the canister surface, both average, $\overline{N u}_{D}$, and local, $N u_{\text {local }}$, values for the Nusselt number were obtained. The effective Nusselt number at each surface temperature node was found as [7],

$$
N u_{\text {eff }}=\left[N u_{D}^{3}+N u_{\text {local }}^{3}\right]^{1 / 3},
$$

where $N u_{\text {local }}$ was calculated based on data presented in [8].

Different correlations have been proposed for the average $N u_{D}$ for free convection on a horizontal cylinder by McAdams [9], Morgan [6] and Churchill [10]. A sensitivity analysis was performed considering all three correlations. It was found that the relation proposed by McAdams performed the best in comparison to published numeric data [2], and the correlation proposed by McAdams is expressed as,

$$
N u_{D}= \begin{cases}0.53 R a_{D}^{0.25} & 10^{4} \leq R a_{D} \leq 10^{9} \\ 0.13 R a_{D}^{0.33} & R a_{D}>10^{9}\end{cases}
$$

where $R a_{D}$ is the Rayleigh number. The expression for $R a_{D}$ is

$$
R a_{D}=\frac{g \beta\left(T_{\mathrm{SS}, \mathrm{o}, i}-T_{\infty}\right) D^{3}}{v \alpha}
$$

where $g$ is the gravitational constant, $\beta$ is the expansion coefficient, which for an ideal gas is unity over the absolute temperature, $v$ is the kinematic viscosity and $\alpha$ is the thermal diffusivity. For simplicity, radiation heat transfer has been neglected within the model.

All aforementioned thermophysical properties are considered temperature-dependent, with the exception of stainless steel and carbon steel, which were taken from [2]. The effective thermal conductivity of the fuel assemblies was modeled using methods typical of spent fuel thermal evaluations [5] and expressed as

$$
\begin{aligned}
k_{\mathrm{eff}}= & 0.3940+1.334 \cdot 10^{-3} T_{m}+2.849 \cdot 10^{-6} T_{m}^{2}+\ldots \\
& +8.359 \cdot 10^{-10} T_{m}^{3}
\end{aligned}
$$

where $T_{m}$ is the mean temperature within an assembly. Thermal Conductivity of the helium back-fill gas, $k_{\mathrm{He}}$, is interpolated linearly at each iteration as a function of temperature based on values presented in [6]. The thermal conductivity, thermal diffusivity and kinematic viscosity of air were interpolated linearly at each iteration as a function of temperature based on values presented in [7].

Since temperature dependent material properties were considered, an iterative process was employed to simultaneously solve the system of equations. The residual for each nodal temperature value and heat through each thermal resistance segment was set to $1 \mathrm{e}-10$.

\section{RESULTS AND DISCUSSION}

The resulting internal and surface temperature distributions generated through the TRN were compared to those obtained numerically through the use of the finite volume method implemented in STAR-CCM [2]. Table II provides measured, numerically- and analytically-predicted surface temperature values, as well as the percent differences between measured 
TABLE II. Temperatures and Model Comparisons. All temperatures are reported in Kelvin.

\begin{tabular}{|lc|cccc|cccc|}
\hline & & \multicolumn{4}{|c|}{ Helium Free-Convection } & \multicolumn{4}{c|}{ Conduction-Only } \\
\hline Location & Measured [2] & {$[2]$} & \% Diff. & TRN & \% Diff. & [2] & \% Diff. & TRN & \% Diff. \\
\hline North $\left(90^{\circ}\right)$ & 321 & 345 & 9.8 & - & - & 346 & 7.5 & 335 & 4.3 \\
East $\left(0^{\circ}\right)$ & 315 & 331 & 5.0 & - & - & 336 & 6.5 & 332 & 5.4 \\
Southeast $\left(330^{\circ}\right)$ & 314 & 316 & 0.6 & - & - & 330 & 5.0 & 340 & 8.0 \\
West $\left(180^{\circ}\right)$ & 315 & 331 & 5.0 & - & - & 334 & 5.9 & 329 & 4.3 \\
Southwest $\left(270^{\circ}\right)$ & 315 & 316 & 0.3 & - & - & 329 & 4.3 & 338 & 7.0 \\
\hline
\end{tabular}

and model values. The numeric models obtained in [2] consider both free convection within the helium back-fill as well as conduction-only cases. Figure 2 shows the predicted internal temperature distribution within the fuel assemblies, helium back-fill, and within and along the stainless steel canister predicted by the TRN. Within the previously published study, surface temperature measurements were collected at five locations on the DCS.

The surface temperature values predicted by the TRN are in good agreement with both the experimentally measured values and those predicted by a full-fledged three-dimensional finite-volume model. Additionally, the TRN model predicts a maximum fuel temperature of 421 [K], whereas [2] obtained a maximum internal temperature of 407 [K]; a $3.4 \%$ difference.

As predicted by Suffield [2], the inclusion of free convection within the helium domain serves primarily to migrate more of the thermal mass toward the top of the canister; it does not have a dramatic effect on the maximum fuel temperature.

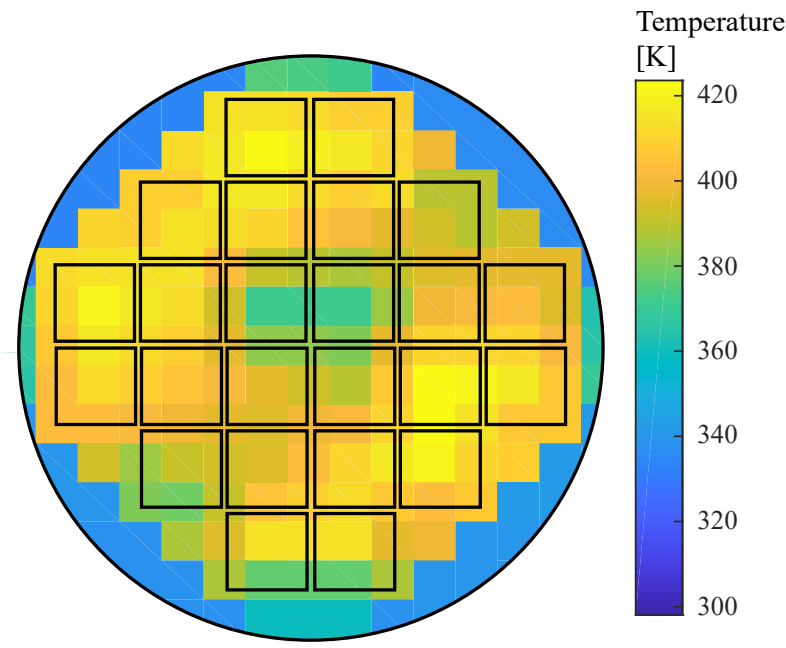

Fig. 2. Predicted internal temperatures within NUHOMS HSM-1 DCS.

\section{CONCLUSIONS}

This study clearly shows that an analytic solution algorithm can be used to accurately predict temperature distributions within the DCS that are in agreement with previously published numeric data. Due to the low computational cost, large numbers of individual spent nuclear fuel modules can now be rapidly analyzed, giving accurate predictions of spatial temperature distributions at various instances in time.

\section{REFERENCES}

1. B. HANSON, H. ALSAED, C. STOCKMAN, D. ENOS, R. MEYER, and K. SORENSON, "Gap analysis to support extended storage of used nuclear fuel, Rev. 0," Tech. rep., Pacific Northwest National Lab.(PNNL), Richland, WA (United States); Idaho ... (2012).

2. S. R. SUFFIELD, J. A. FORT, H. E. ADKINS, J. M. CUTA, B. A. COLLINS, and E. R. SICILIANO, "Thermal modeling of NUHOMS HSM-15 and HSM-1 storage modules at Calvert Cliffs nuclear power station ISFSI," Tech. rep., Pacific Northwest National Lab.(PNNL), Richland, WA (United States) (2012).

3. J. A. FORT, T. E. MICHENER, S. R. SUFFIELD, and D. J. RICHMOND, "Thermal Modeling of a Loaded Magnastor Storage System at Catawba Nuclear Station,” Tech. rep., Pacific Northwest National Lab.(PNNL), Richland, WA (United States) (2016).

4. R. POŠKAS, V. ŠIMONIS, P. POŠKAS, and A. SIRVYDAS, "Thermal analysis of CASTOR RBMK-1500 casks during long-term storage of spent nuclear fuel," Annals of Nuclear Energy, 99, 40-46 (2017).

5. R. BAHNEY and T. LOTZ, "Spent nuclear fuel effective thermal conductivity report," Prepared for the US DOE, Yucca Mountain Site Characterization Project Office by TRW Environmental Safety Systems, Inc., July, 11 (1996).

6. V. D. ARP and R. D. MCCARTY, "Thermophysical Properties of Helium-4 from 0.8 to $1500 \mathrm{~K}$ with Pressures to 2000 MPa," Tech. rep., United States Deparmtent of Commerce, Technology Administration, National Institute of Standards and Technology (1989).

7. F. P. INCROPERA, A. S. LAVINE, T. L. BERGMAN, and D. P. DEWITT, Fundamentals of heat and mass transfer, Wiley (2007).

8. S. KAKAÇ, R. K. SHAH, and W. AUNG, "Handbook of single-phase convective heat transfer," (1987).

9. W. MCADAMS, Heat Transmission, McGraw Hill (1954).

10. S. W. CHURCHILL and H. H. CHU, "Correlating equations for laminar and turbulent free convection from a vertical plate," International journal of heat and mass transfer, 18, 11, 1323-1329 (1975). 